\title{
A surface energy model and application to mechanical behavior analysis of single crystals at sub-micron scale
}

\author{
X.L. Chen, H.S. Ma, L.H. Liang, Y.G. Wei * \\ LNM, Institute of Mechanics, Chinese Academy of Sciences, Beijing 100080, China
}

\section{A R T I C L E I N F O}

\section{Article history:}

Received 14 November 2008

Received in revised form 3 March 2009

Accepted 13 March 2009

Available online 17 April 2009

\section{Keywords:}

Size effect

Surface energy

Elastic-plastic analysis

Single crystals

\begin{abstract}
A B S T R A C T
Size effects of mechanical behaviors of materials are referred to the variation of the mechanical behavior due to the sample sizes changing from macroscale to micro-/nanoscales. At the micro-/nanoscale, since sample has a relatively high specific surface area (SSA) (ratio of surface area to volume), the surface energy effect, although it is often neglected at the macroscale, becomes prominent in governing the mechanical behavior. In the present research, a continuum model considering the surface energy effect is developed through introducing the surface energy to total potential energy. Simultaneously, a corresponding finite element method is developed. The model is used to analyze the axial equilibrium strain problem for a $\mathrm{Cu}$ nanowire at the external loading-free state. As another application of the model, from dimensional analysis, the size effects of uniform compression tests on the microscale cylinder specimens for $\mathrm{Ni}$ and Au single crystals are analyzed and compared with experiments in literatures.
\end{abstract}

(c) 2009 Elsevier B.V. All rights reserved.

\section{Introduction}

Recent researches have shown that the micro-/nanostructured materials or materials at the micro-/nanoscale possess the superior mechanical, physical and chemical properties $[7,9,18]$. At the micro-/nanoscale, size effects of mechanical behavior often become prominent, and they are usually considered as a result from imposed deformation gradients in crystalline materials $[8,28,15]$ etc. Recently, several studies emphasize that at the micro-/nanoscale, the sample geometry size affects the mechanisms of deformation and strength. Using molecular dynamics simulations for simple shear of Ni single crystals, Horstemeyer et al. [14] found that the yield strength depends on the specific surface area (SSA) of the sample without considering strain gradient effects. Microscale cylindrical pillars were tested under the uniaxial compression for pure $\mathrm{Ni}$ and Ni-base alloys [25,4] and for Au single crystals $[11,26]$. The stress-strain curves showed dramatic size effects when sample dimensions are at the micro- or sub-micron scale. Samples were machined from a bulk single crystal by focused ion beam milling, and hence they are considered to have identical initial internal structures. Size effects are mainly induced by the variation of sample geometric sizes.

At the micro-/nanoscale, since material has a relatively high SSA, the surface energy effect becomes prominent. The surface stress effects on size-dependent elastic properties of nanoscale structures have been studied widely $[23,24,29,5,6,12,10]$ etc. For example, Gao et al. [10] developed a finite element method to com-

\footnotetext{
* Corresponding author. Tel.: +86 1082543956; fax: +86 1062561284

E-mail address: Ywei@LNM.imech.ac.cn (Y.G. Wei).
}

pute the surface energy effect which was described by adopting a set of surface elastic constitutive equations of Miller and Shenoy [20] within a surface layer and leading to introduce the surface stress and strain components. However, it is difficult to measure the surface stress tensor of solids experimentally, and it is still necessary to adopt the atomistic simulations to calculate surface stresses or determine the surface elastic constants. Recently Park et al. [21] presented a surface Cauchy-Born model to study surface effects while the homogeneous deformation assumption is required.

In this paper, the surface energy effect is still expected to be prominent in governing size effect on the mechanical behavior of single crystals, and the surface energy density $(\gamma)$ is still assumed a material constant [30] within a length scale region from sub-micron to macroscale. Based on the assumption, a continuum mechanics model considering the surface energy effect will be developed. A new finite element method corresponding the new model is also developed for the model application. The model will be used to analyze the axial equilibrium strain variation of a $\mathrm{Cu}$ nanowire. Based on the modified continuum model, size effects of compression tests on the sub-micron scale cylinder specimens of $\mathrm{Ni}$ and $\mathrm{Au}$ single crystals are analyzed and compared with experimental measurements in literatures.

\section{Quantity level analysis of surface energy effect}

Geometrically, the specific surface area $(S / V)$ of a sample is a function of sample sizes,

$\frac{S}{V}=f\left(L, L_{1}, L_{2}, \ldots\right)$. 
From dimensional analysis, Eq. (1) can be written as a nondimensional relation

$L \cdot \frac{S}{V}=\pi\left(\frac{L_{1}}{L}, \frac{L_{2}}{L}, \ldots\right)$.

From Eq. (2), the SSA can be expressed by a characteristic length $(L)$ and several normalized shape parameters. SSA relations for three typical samples are shown in Fig. 1. From Eq. (2) and Fig. 1, the relation between the SSA and characteristic length for a spherical or a cubic sample is linear in the logarithmic coordinate system, as shown in Fig. 2. It can be seen that $S / V$ is of order $10^{8} \mathrm{~m}^{-1}$ at the sub-micron scale approximately, while it is of order $10^{2} \mathrm{~m}^{-1}$ at the macroscale. For the typical metal materials, the quantity levels of the stress $(\sigma)$, strain $(\varepsilon)$, strain energy density $\left(U_{0}\right)$ and surface energy density $(\gamma)$ are given as follows

$\sigma \sim 10^{1}-10^{3} \mathrm{MPa} \quad \varepsilon \sim 10^{-2}-10^{-1}$,

$U_{0} \sim \sigma \cdot \varepsilon / 2 \sim 10^{5}-10^{8} \mathrm{~J} / \mathrm{m}^{3}, \quad \gamma \sim 1 \mathrm{~J} / \mathrm{m}^{2}$.

Therefore, the order of the ratio (surface energy to strain energy) is given as

$\frac{\gamma S}{U_{0} V} \sim 10^{-6}-10^{-3}$

at the macroscale, and

$\frac{\gamma S}{U_{0} V} \sim\left(1-10^{3}\right)$

at the micro-/nanoscale.

Both Eqs. (4) and (5) imply that surface energy effect can be rationally ignored at the macroscale while it should be considered at the micro-/nanoscale.

\begin{tabular}{|c|c|c|}
\hline Sphere & Cylinder & Cuboid \\
\hline$L \cdot \frac{S}{V}=6$ & $L \cdot \frac{S}{V}=2 \cdot\left(2+\frac{L}{L_{1}}\right)$ & $L \cdot L_{1}$ \\
\hline & $L$ & \\
\hline
\end{tabular}

Fig. 1. Relations of specific surface area with characteristic size for three typical samples.

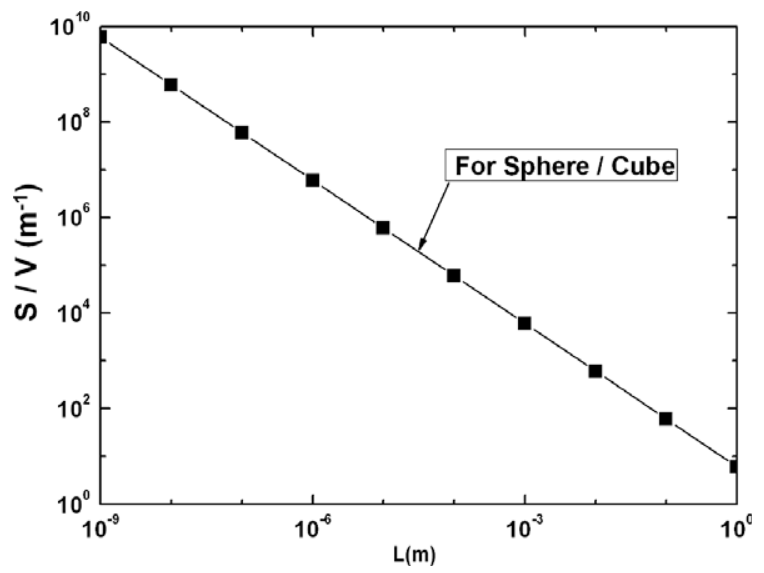

Fig. 2. Quantity level variation of specific surface area $(S / V)$ with characteristic size of samples.

\section{A surface energy model and corresponding finite element method}

According to analysis in the last section, the surface energy effect should be considered when sample sizes are at the micro-/ nanoscale. The conventional minimum potential principle will be revised to include the surface energy effect. In the new model, the total potential energy of solid $(\Pi)$ is consisted of three parts, the strain energy $\left(U^{b}\right)$, surface energy $\left(U^{s}\right)$ and external force potential $(-W)$. The total potential energy can be expressed as

$\Pi=U^{b}+U^{s}-W$

In Eq. (6), $U^{b}$ and $-W$ can be expressed as usual

$U^{b}=\int_{V} U_{0} d V$

$-W=-\int_{V} \overline{f_{i}} u_{i} d V-\int_{S_{\sigma}} \overline{t_{i}} u_{i} d S$

where $U_{0}$ is strain energy density, $\bar{f}_{i}$ and $\overline{t_{i}}(i=1,2,3)$ are body force and applied surface traction, respectively.

The surface energy $\left(U^{s}\right)$ can be calculated by

$U^{s}=\int_{S^{\prime}} \gamma d S^{\prime}$

where $S^{\prime}$ is the deformed surface area, which can be related with initial surface area $(S)$ by

$d S^{\prime} \approx\left[1+l_{i} l_{j} \varepsilon_{i j}+m_{i} m_{j} \varepsilon_{i j}+l_{i} l_{j} \varepsilon_{i j} m_{p} m_{q} \varepsilon_{p q}-\left(l_{i} m_{j} \varepsilon_{i j}\right)^{2} / 2\right] \cdot d S$,

from deformation geometrical analysis, where $\varepsilon_{i j}(i, j=1,2,3)$ are the surface boundary strain components in global strain field, which are different from that of the surface strains within a surface layer specially defined by Miller and Shenoy [20] by presenting a set of surface constitutive equations; $\boldsymbol{l}$ and $\boldsymbol{m}$ are the orthogonal unit vectors on surface of solid, which are expressed as

$\boldsymbol{l}=\left[l_{1} l_{2} l_{3}\right]^{\mathrm{T}} \quad \boldsymbol{m}=\left[m_{1} m_{2} m_{3}\right]^{\mathrm{T}}$,

$\left(\boldsymbol{l}_{i}, m_{i}\right)(i=1,2,3)$ are the direction cosines of $\boldsymbol{l}$ and $\boldsymbol{m}$ relative to the global coordinate system.

From Eqs. (6)-(9), (1), the variational relation of total system can be expressed as

$\int_{V} \delta \boldsymbol{\varepsilon}^{\mathrm{T}} \boldsymbol{\sigma} d V+\int_{S} \delta \boldsymbol{\varepsilon}^{\mathrm{T}}\left(\boldsymbol{R}+\boldsymbol{D}_{s} \boldsymbol{\varepsilon}\right) \gamma d S-\left(\int_{V} \delta \boldsymbol{u}^{\mathrm{T}} \overline{\boldsymbol{f}} d S+\int_{S_{\sigma}} \delta \boldsymbol{u}^{\mathrm{T}} \overline{\boldsymbol{t}} d S\right)=0$,

where $\varepsilon$ and $\sigma$ are the strain vector and stress vector respectively, defined as

$\boldsymbol{\varepsilon}=\left[\begin{array}{lllll}\varepsilon_{x} \varepsilon_{y} & \varepsilon_{z} & \gamma_{x y} & \gamma_{y z} & \gamma_{z x}\end{array}\right]^{\mathrm{T}}$

$\boldsymbol{\sigma}=\frac{\partial U_{0}}{\partial \varepsilon}=\boldsymbol{D}_{\mathrm{ep}} \boldsymbol{\varepsilon}$

$\boldsymbol{D}_{\mathrm{ep}}$ is elastic-plastic matrix. In Eq. (2), matrix $\boldsymbol{R}$ and matrix $\boldsymbol{D}_{\mathrm{s}}$ can be expressed as

$\boldsymbol{R}=\boldsymbol{R}_{1}+\boldsymbol{R}_{2}, \quad D_{s}=\boldsymbol{R}_{1} \boldsymbol{R}_{2}^{\mathrm{T}}+\boldsymbol{R}_{2} \boldsymbol{R}_{1}^{\mathrm{T}}-\boldsymbol{R}_{3} \boldsymbol{R}_{3}^{\mathrm{T}}$
$\boldsymbol{R}_{1}=\left[l_{2}^{1} l_{2}^{2} l_{2}^{3} l_{1} l_{2} l_{2} l_{3} l_{3} l_{1}\right]^{\mathrm{T}}$
$\boldsymbol{R}_{2}=\left[m_{1}^{2} m_{2}^{2} m_{3}^{2} m_{1} m_{2} m_{2} m_{3} m_{3} m_{1}\right]^{\mathrm{T}}$
$\boldsymbol{R}_{3}=\left[2 l_{1} m_{1} 2 l_{2} m_{2} 2 l_{3} m_{3} l_{1} m_{2}+l_{2} m_{1} l_{2} m_{3}+l_{3} m_{2} l_{3} m_{1}+l_{1} m_{3}\right]^{\mathrm{T}}$

From Eqs. (2)-(4), one can obtain

$\boldsymbol{K a}=\boldsymbol{P}$

where $\boldsymbol{a}$ is the node displacement vector of the total system; stiffness matrix and node force vector can be expressed as 


$$
\begin{aligned}
& \boldsymbol{K}=\sum_{e} \boldsymbol{G}_{e}^{\mathrm{T}} \boldsymbol{K}^{e} \boldsymbol{G}_{e}, \quad \boldsymbol{K}^{e}=\boldsymbol{K}_{b}^{e}+\boldsymbol{K}_{s}^{e} \\
& \boldsymbol{P}=\sum_{e} \boldsymbol{G}_{e}^{\mathrm{T}} \boldsymbol{P}^{e}, \quad \boldsymbol{P}^{e}=\boldsymbol{P}_{b}^{e}+\boldsymbol{P}_{s}^{e}+\boldsymbol{P}_{\gamma}^{e}
\end{aligned}
$$

$\boldsymbol{G}_{e}$ is the connectivity matrix [1] by which the element displacement matrix can be transformed into $\boldsymbol{a} ; \boldsymbol{K}_{b}^{e}$ and $\boldsymbol{K}_{s}^{e}$ are stiffness matrixes of the bulk element and surface element respectively. The equivalent nodal force matrixes of elements, $\boldsymbol{P}_{b}^{e} \boldsymbol{P}_{s}^{e}$ and $\boldsymbol{P}_{\gamma}^{e}$, are calculated through the known body forces, surface tractions and surface energy respectively. $\boldsymbol{K}_{b}^{e}, \boldsymbol{K}_{s}^{e}, \boldsymbol{P}_{b}^{e}, \boldsymbol{P}_{s}^{e}$ and $\boldsymbol{P}_{\gamma}^{e}$ can be expressed as

$\boldsymbol{K}_{b}^{e}=\int_{V^{e}} \boldsymbol{B}_{b}^{\mathrm{T}} \boldsymbol{D}_{\mathrm{ep}} \boldsymbol{B}_{b} d V, \quad \boldsymbol{K}_{s}^{e}=\int_{S^{e}} \boldsymbol{B}_{s}^{\mathrm{T}} \boldsymbol{D}_{s} \boldsymbol{B}_{s} \gamma d S$,

$\boldsymbol{P}_{b}^{e}=\int_{V^{e}} \boldsymbol{N}^{\mathrm{T}} \overline{\boldsymbol{f}} d V, \quad \boldsymbol{P}_{s}^{e}=\int_{S_{\sigma}^{e}} \boldsymbol{N}^{\mathrm{T}} \overline{\boldsymbol{t}} d S, \quad \boldsymbol{P}_{\gamma}^{e}=-\int_{S^{e}} \boldsymbol{B}_{s}^{\mathrm{T}} \boldsymbol{R} \gamma d S$,

where $\boldsymbol{B}_{b}$ and $\boldsymbol{B}_{s}$ are the strain matrixes of the bulk element and surface element respectively, and $(\boldsymbol{N})$ is the matrix of shape functions.

In our finite element method considering the surface energy effect, the 20-node hexahedral brick isoparametric element is chosen as the bulk elements. It is worth noting that a surface element corresponds to a face of the bulk element, with 8 nodes. For the 20 node hexahedral brick isoparametric element, two unit vectors $l$ and $\boldsymbol{m}$ given in Eq. (1), which describe the orientation of the surface element, can be expressed by the Gram-Schmidt orthogonalization in parametrical coordinates $(\xi, \eta, \zeta)$ as

$\boldsymbol{l}=\frac{\boldsymbol{r}_{\zeta}}{\left|\boldsymbol{r}_{\zeta}\right|} \quad$ and $\quad \boldsymbol{m}=\frac{\boldsymbol{r}_{\xi}-\left(\boldsymbol{r}_{\xi} \cdot \boldsymbol{l}\right) \boldsymbol{l}}{\left|\boldsymbol{r}_{\xi}-\left(\boldsymbol{r}_{\xi} \cdot \boldsymbol{l}\right) \boldsymbol{l}\right|} \quad$ (for surface elements with $\eta= \pm 1$ )

$\boldsymbol{l}=\frac{\boldsymbol{r}_{\xi}}{\left|\boldsymbol{r}_{\xi}\right|} \quad$ and $\quad \boldsymbol{m}=\frac{\boldsymbol{r}_{\eta}-\left(\boldsymbol{r}_{\eta} \cdot \boldsymbol{l}\right) \boldsymbol{l}}{\left|\boldsymbol{l}_{\eta}-\left(\boldsymbol{r}_{\eta} \cdot \boldsymbol{l}\right) \boldsymbol{l}\right|} \quad$ (for surface elements with $\zeta= \pm 1$ )

$\boldsymbol{l}=\frac{\boldsymbol{r}_{\eta}}{\left|\boldsymbol{r}_{\eta}\right|} \quad$ and $\quad \boldsymbol{m}=\frac{\boldsymbol{r}_{\zeta}-\left(\boldsymbol{r}_{\zeta} \cdot \boldsymbol{l}\right) \boldsymbol{l}}{\left|\boldsymbol{r}_{\zeta}-\left(\boldsymbol{r}_{\zeta} \cdot \boldsymbol{l}\right) \boldsymbol{l}\right|} \quad$ (for surface elements with $\xi= \pm 1$ )

where $\boldsymbol{r}_{\xi}, \boldsymbol{r}_{\eta}$, and $\boldsymbol{r}_{\zeta}$ are defined by global coordinates $(x, y, z)$ and parametrical coordinates $(\xi, \eta, \zeta)$ as

$\boldsymbol{r}_{\xi}=\left[\frac{\partial x}{\partial \xi} \frac{\partial y}{\partial \xi} \frac{\partial z}{\partial \xi}\right]^{\mathrm{T}}, \boldsymbol{r}_{\eta}=\left[\frac{\partial x}{\partial \eta} \frac{\partial y}{\partial \eta} \frac{\partial z}{\partial \eta}\right]^{\mathrm{T}}, \boldsymbol{r}_{\zeta}=\left[\frac{\partial x}{\partial \zeta} \frac{\partial y}{\partial \zeta} \frac{\partial z}{\partial \zeta}\right]^{\mathrm{T}}$

From Eqs. (4), (6), (7), (8), (9), the equilibrium Eq. (5) can be expressed completely. It should be noted that the global stiffness matrix, $K$ in Eq. (5), is positive definite after the treatment of constraints in the traditional finite method [19]. However, $\boldsymbol{K}$ is generally a sparse, symmetrical but non-positive definite matrix in the present model considering the surface energy effect, so some usual methods such as Cholesky decomposition can not be used to solve Eq. (5).

An elastic-plastic material with power-law hardening feature is considered here,

$\frac{\sigma_{e}}{\sigma_{Y}}= \begin{cases}\varepsilon_{e} / \varepsilon_{Y} & \left(\sigma_{e} \leqslant \sigma_{Y}\right), \\ \left(\varepsilon_{e} / \varepsilon_{Y}\right)^{N} & \left(\sigma_{e}>\sigma_{Y}\right),\end{cases}$

where $\sigma_{e}$ and $\varepsilon_{e}$ are the Von Mises effective stress and effective strain respectively, $N$ is the strain-hardening exponent; $\sigma_{Y}$ is the initial yield stress at the macroscale, and $\varepsilon_{Y}$ is the corresponding yield strain, $\varepsilon_{Y}=\sigma_{Y} / E$, where $E$ is the macroscale Young's modulus.

\section{Applications of the surface energy model}

\subsection{Free relaxation of external loading-free nanowires}

Metal nanowires have an interesting mechanical property. By using atomistic simulations, Diao et al. ${ }^{* *}[2,3]$ found that for an external loading-free Au nanowire, the compressive residue stress always exists in the interior of the nanowire, and the $<100>$ nanowire will undergo a phase transformation process from face-cen- tered-cubic (FCC) structure to body-centered-tetragonal (BCT) structure when nanowire size (width of square cross-section) is less than $2 \mathrm{~nm}$. By molecular dynamics simulations, Liang and Zhou [17] observed that for $\mathrm{Cu}$ nanowire when cross-section size is smaller than $2.17 \times 2.17 \mathrm{~nm}$, spontaneous lattice was reoriented with the cross-sectional shape changing from square to rhombic, and the wire decreased in length by $29.3 \%$ at a temperature of $300 \mathrm{~K}$, approximately.

From the present surface energy model, for the external loading-free nanowire case, due to the surface energy effect, the tension stress is formed on the surface, which is balanced by the compressive stress in the interior of nanowire. At the self-balance state, generally the length of the nanowire will be shortening due to the interior compression, which can be determined by calculating the axial equilibrium strain. In this subsection, our surface energy model will be used to investigate the free relaxation of single crystalline $\mathrm{Cu}$ nanowires and calculate the axial equilibrium strain. Initial length of the nanowire is $L_{1}$ and the size of square cross-section is $L$. Assume that the surface energy density is a material constant at the sub-micron scale. Both 3-D elastic-plastic and 1-D elastic analyses are performed.

For the external loading-free nanowires using the 1-D elastic analysis, Eq. (6) can be simplified as a theoretical formulation,

$\Pi=E \varepsilon^{2} L^{2} L_{1} / 2+\gamma\left[2 L^{2}(1-v \varepsilon)^{2}+4 L L_{1}(1-v \varepsilon)(1+\varepsilon)\right]$,

where $\varepsilon$ is axial equilibrium strain of nanowire, $v$ is Poisson's ratio. The axial equilibrium strain with respect to the nanowire size and the surface energy density can be found from the functional stationary condition $(\delta \Pi=0)$ to $\varepsilon$, one have

$\varepsilon=\frac{4 v-4(1-v) \cdot\left(L_{1} / L\right)}{(E / \gamma) \cdot L \cdot\left(L_{1} / L\right)+4 v^{2}-8 v \cdot\left(L_{1} / L\right)}$

The 3-D elastic-plastic analysis model has been depicted in detail in the last section. In the numerical examples, for the $\mathrm{Cu}$ single crystals, material parameters are taken as: $E=200 \mathrm{GPa}, v=0.3$, $N=0.2, \sigma_{Y}=300 \mathrm{MPa}, \gamma=1.725 \mathrm{~J} / \mathrm{m}^{2}$, where the value of surface energy density used in this study is come from Hirth and Lothe [13]. The finite element method developed in last section is used. In the present calculations, the 20-node hexahedral brick isoparametric element is chosen as the bulk elements, and the surface element corresponds to a face of the bulk element, with 8 nodes. The cuboid element is used and the mesh is divided along the sample length direction and cross-section by $50 \times 3 \times 3$. Total element number is 450 . For each element, 27 Gauss points are adopted in the integration. For the given surface energy density, the displacement field is computed, so that the axial equilibrium strain can be obtained.

The simulation results based on both 1-D and 3-D surface energy model are compared with the results of molecular static (MS) results given by Liang et al. [16], as shown in Fig. 3. All the results show nanowires contraction in their length direction. With decreasing the size of cross-section, the axial equilibrium strain (contraction) increases. From the results of 3-D surface energy model, long and slender nanowires contract more than short and thick nanowires, which are consistent with the conclusion based on the surface Cauchy-Born model [22].

\subsection{Size effects of flow stress in compressive tests for single crystalline samples}

Both surface and volume effects affect the flow stress of single crystalline materials at the micro- or sub-micron scale $[11,4]$. According to the present surface energy model given in the last section, we have an additional material parameter, surface energy density $\gamma$. Referring to the dimensional analysis method using by 


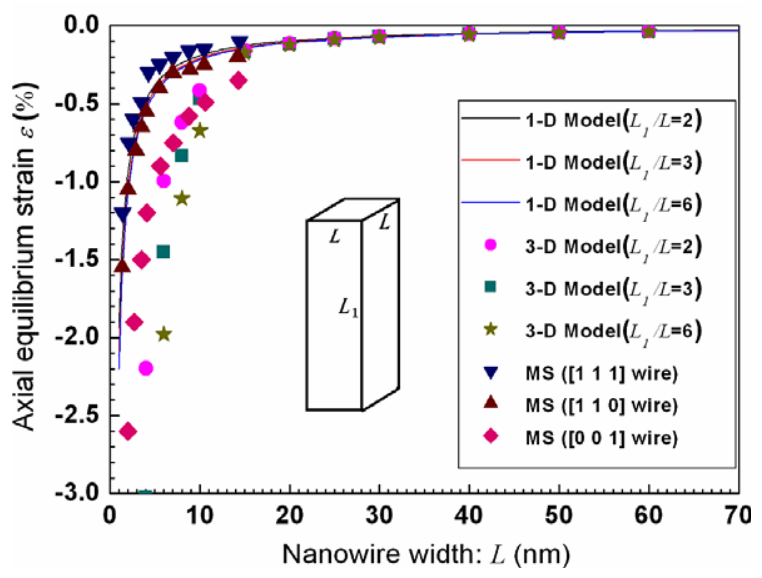

Fig. 3. Axial equilibrium strain as a function of the size of single crystalline $\mathrm{Cu}$ nanowire with square cross-section is shown, using 1-D model Eq. (18) and 3-D model (finite element method developed in the present paper). The results of molecular static simulations (MS) with three orientations [16] are also shown.

Zheng [30] who analyzed fracture strength of solid considering the surface energy effect, we can easily develop a relationship between flow stress and the surface energy density. The flow stress $\left(\sigma_{s}\right)$ of single crystal samples can be described by two types of independent dimensionless parameters (taking a cylinder sample as example here)

$\frac{\sigma_{s}}{\sigma_{Y}}=\pi_{f}\left(\frac{\gamma}{\sigma_{Y} L}, \frac{L_{1}}{L}\right)$,

where the dimensionless parameter, $\gamma /\left(\sigma_{Y} L\right)$ describes a ratio of surface energy to volume deformation energy for the uniform loading sample, another parameter $L_{1} / L$ is the aspect ratio, $\sigma_{Y}$ is the flow stress of material at the case of macroscale sample size. The order of magnitude analysis about the surface energy effect is given in Table 1. From Table 1, it is clear that for the elastic-plastic case, the surface energy effect is considerably large at the nanometer scale. When Eq. (13) is expanded into a Taylor series, and the first-order quantity in the series is kept, one can easily obtain a linear relation between the normalized flow stresses and the normalized surface energy density as,

$\frac{\sigma_{s}}{\sigma_{Y}}=1+\frac{\gamma}{\sigma_{Y} L} f_{1}\left(\frac{L_{1}}{L}\right)$

and can be compared with the recent experimental results given by Greer et al. [11] and Dimiduk et al. [4], where the undetermined function $f_{1}$ is only dependent on the sample aspect ratio.

Similarly, for an elastic case, one can obtain a concise relation of Young's modulus $\left(E_{g}\right)$ with surface energy density based on the present surface energy model through dimensional analysis,

$\frac{E_{g}}{E}=1+\frac{\gamma}{E L} \psi_{1}\left(\frac{L_{1}}{L}\right)$

where $E$ is the Young's modulus at the case of macroscale sample size. The expression (15) is similar to that given by Wang et al. [27].

Table 1

Order of magnitude analysis of surface energy effect and influence on the flow stress for elastic-plastic materials and on the Young's modulus for elastic materials.

\begin{tabular}{llllll}
\hline$L$ & $1 \mathrm{~m}$ & $1 \mathrm{~mm}$ & $1 \mu \mathrm{m}$ & $10 \mathrm{~nm}$ & $1 \mathrm{~nm}$ \\
\hline$\frac{\gamma}{\sigma_{\gamma} L}$ & $10^{-8}$ & $10^{-5}$ & $10^{-2}$ & $10^{0}$ & $10^{1}$ \\
$\frac{\gamma}{E L}$ & $10^{-11}$ & $10^{-8}$ & $10^{-5}$ & $10^{-3}$ & $10^{-2}$ \\
\hline$\gamma \sim 1 \mathrm{Jm}^{-2}, \sigma_{\gamma} \sim 10^{2} \mathrm{MPa}$, and $E \sim 10^{2} \mathrm{GPa}$. & & &
\end{tabular}

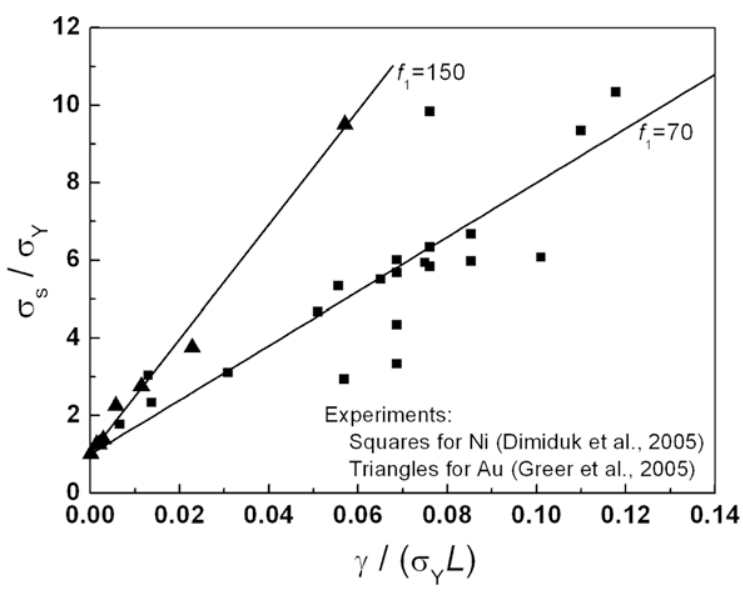

Fig. 4. Variation of material flow stress with surface energy density. Experimental results can be modeled effectively using the theoretical model.

The order of magnitude analysis about the surface energy effects on the flow stress and Young's modulus is given in Table 1. From Table $1, \gamma /\left(\sigma_{Y} L\right)$ is of order $10^{-2}$ at the micrometer scale. However, $\gamma /(E L)$ is of order $10^{-2}$ at the nanometer scale. This indicates that for plastic case the size effects are usually prominent starting from microscale to smaller scale, while for elastic case they are prominent starting from nanometer scale to much smaller scale. It is interesting to note that the intrinsic length scale $\gamma / E$ can be traced back to the classical Griffith theory for brittle fracture [27].

In Fig. 4, the above linear relation Eq. (14) is used to describe the experimental results. The experimental data are from microscale uniaxial compression tests. From Fig. 4, one can find that this simple relation can describe the size effects of the flow stress of single crystals effectively.

\section{Concluding remarks}

In the present research, through the order of magnitude analysis for the SSA, a new model considering surface energy effect has been developed. The corresponding finite element method has also been developed. The surface energy model has been used to analyze the axial equilibrium strain variations for the external loading-free nanowires. Based on the surface energy model, the size effects of flow stress and Young's modulus have been analyzed from dimensional analysis, and the analysis results are used to successfully describe the experimental data. The order of magnitude analysis for the surface energy density and its influence on the flow stress has been performed. The main results obtained in the present research can be summarized to the following key points: (1) as a length scale parameter, the SSA of sample undergoes a huge increase when sample size changes from the macroscale to micro-/ nanoscale, so that the surface energy effect becomes obvious, especially when sample size reaches at the micro-/nanoscale. (2) Taking the surface energy density as a material parameter, a surface energy model has been developed through introducing the surface energy density to the conventional continuum theory. The corresponding finite element method has been developed. Through analyzing a typical nanoscale problem, the relaxation of external loading-free nanowire, the effectiveness of the model has been testified. (3) Through the order of magnitude analysis for the surface energy effect and for its influence on the flow stress and Young's modulus, we have found that for a plastic problem the size effects are usually prominent starting from microscale to smaller scale, while for an elastic problem they are prominent starting from nanometer scale to much smaller scale. 
It is worth noting that in the present research, about size effects of mechanical behavior, although some important features can be described and captured by using the surface energy model, however, the surface energy density is assumed as a material constant and only single crystal materials are discussed. Generally speaking, the surface energy density should be a variable as a function of SSA surface deformation or surface temperature etc. Further and indepth research work on surface energy effects is still needed. On the other hand, the size effects should be dependent on both the surface effect and the volume effect. About the volume effect, the strain gradient effect should be important at the micro-/nanoscale, which is not considered in the present model.

\section{Acknowledgements}

The subject is supported by the National Science Foundation of China through Grants Nos. 10672163, 10721202, 10802088 and 90816004 and by the Chinese Academy of Science through Grant KJCX-YW-M04.

\section{References}

[1] T. Belytschko, W.K. Liu, B. Moran, Nonlinear Finite Elements for Continua and Structures, John Wiley and Sons, 2000. p. 39

[2] J. Diao, K. Gall, M.L. Dunn, Nat. Mater. 2 (2003) 656-660.

[3] J. Diao, K. Gall, M.L. Dunn, J.A. Zimmerman, Acta Mater. 54 (2006) 643-653.

[4] D.M. Dimiduk, M.D. Uchic, T.A. Parthasarathy, Acta Mater. 53 (2005) 40654077.
[5] R. Dingreville, J. Qu, M. Cherkaoui, J. Mech. Phys. Solids 53 (2005) 1827-1854.

[6] H.L. Duan, J. Wang, B.L. Karihaloo, Z.P. Huang, Acta Mater. 54 (2006) 29832990.

[7] X.F. Duan, Y. Huang, Y. Cui, J.F. Wang, C.M. Lieber, Nature 409 (2001) 66-69.

[8] N.A. Fleck, J.W. Hutchinson, J. Mech. Phys. Solids 49 (2001) 2245-2271.

[9] R.S. Friedman, M.C. McAlpine, D.S. Ricketts, D. Ham, C.M. Lieber, Nature 434 (2005) 1085

[10] W. Gao, S.W. Yu, G.Y. Huang, Nanotechnology 17 (2006) 1118-1122.

[11] J.R. Greer, W.C. Oliver, W.D. Nix, Acta Mater. 53 (2005) 1821-1830.

[12] L.H. He, C.W. Lim, Int. J. Solids Struct. 43 (2006) 132-143.

[13] J.P. Hirth, J. Loethe, Theory of Dislocations, second ed., John Wiley and Sons, 1982. p. 839.

[14] M.F. Horstemeyer, M.I. Baskes, S.J. Plimpton, Acta Mater. 49 (2001) 4363-4374

[15] Y. Huang, S. Qu, K.C. Hwang, M. Li, H. Gao, Int. J. Plasticity 20 (2004) 753-782.

[16] H.Y. Liang, M. Upmanyu, H.C. Huang, Phys. Rev. B 71 (2005) 241403.

[17] W.W. Liang, M. Zhou, J. Eng. Mater. Technol. 127 (2005) 423-433.

[18] C.M. Lieber, Z.L. Wang, MRS Bull. 32 (2007) 99-108.

[19] G.R. Liu, S.S. Quek, The Finite Element Method: A Practical Course, Butterworth-Heinman, Oxford, 2003. p. 57

[20] R.E. Miller, V.B. Shenoy, Nanotechnology 11 (2000) 139-147.

[21] H.S. Park, P.A. Klein, G.J. Wagner, Int. J. Numer. Meth. Eng. 68 (2006) 10721095.

[22] H.S. Park, P.A. Klein, Phys. Rev. B 75 (2007) 085408.

[23] P. Sharma, S. Ganti, N. Bhate, Appl. Phys. Lett. 82 (2003) 535-537.

[24] H.W. Shim, L.G. Zhou, H.C. Huang, T.S. Cale, Appl. Phys. Lett. 86 (2005) 151912.

[25] M.D. Uchic, D.M. Dimiduk, J.N. Florando, W.D. Nix, Science 305 (2004) 986989.

[26] C.A. Volkert, E.T. Lilleodden, Philos. Mag. 86 (2006) 5567-5579.

[27] J. Wang, H.L. Duan, Z.P. Huang, B.L. Karihaloo, Proc. R. Soc. A 462 (2006) 13551363.

[28] Y. Wei, J.W. Hutchinson, J. Mech. Phys. Solids 51 (2003) 2037-2056.

[29] F.Q. Yang, J. Appl. Phys. 95 (2004) 3516-3520.

[30] Z.M. Zheng, Adv. Mech. 12 (1982) 133-140 (in Chinese). 